\title{
Multicolour imaging with spectral photon-counting CT: a phantom study
}

\author{
Salim Si-Mohamed ${ }^{1,2^{*}}$ (D), Daniel Bar-Ness', Monica Sigovan', Valérie Tatard-Leitman', David P. Cormode ${ }^{4}$, \\ Pratap C. Naha ${ }^{4}$, Philippe Coulon ${ }^{5}$, Lucie Rascle ${ }^{1}$, Ewald Roessl ${ }^{6}$, Michal Rokni $^{3}$, Ami Altman $^{3}$, Yoad Yagil ${ }^{3}$, \\ Loic Boussel ${ }^{1,2}$ and Philippe Douek ${ }^{1,2}$
}

\begin{abstract}
Background: To evaluate the feasibility of multicolour quantitative imaging with spectral photon-counting computed tomography (SPCCT) of different mixed contrast agents.

Methods: Phantoms containing eleven tubes with mixtures of varying proportions of two contrast agents (i.e. two selected from gadolinium, iodine or gold nanoparticles) were prepared so that the attenuation of each tube was about $280 \mathrm{HU}$. Scans were acquired at $120 \mathrm{kVp}$ and $100 \mathrm{mAs}$ using a five-bin preclinical SPCCT prototype, generating conventional, water, iodine, gadolinium and gold images. The correlation between prepared and measured concentrations was assessed using linear regression. The cross-contamination was measured for each material as the root mean square error (RMSE) of its concentration in the other material images, where no signal was expected. The contrast-to-noise ratio (CNR) relative to a phosphate buffered saline tube was calculated for each contrast agent.

Results: The solutions had similar attenuations $(279 \pm 10 \mathrm{HU}$, mean \pm standard deviation) and could not be differentiated on conventional images. However, a distinction was observed in the material images within the same samples, and the measured and prepared concentrations were strongly correlated $\left(R^{2} \geq 0.97,0.81 \leq\right.$ slope $\leq 0.95,-0.68 \leq$ offset $\leq 0.89 \mathrm{mg} / \mathrm{mL}$ ). Cross-contamination in the iodine images for the mixture of gold and gadolinium contrast agents (RMSE $=0.34 \mathrm{mg} / \mathrm{mL}$ ) was observed. CNR for $1 \mathrm{mg} / \mathrm{mL}$ of contrast agent was better for the mixture of iodine and gadolinium $\left(C N R_{\text {iodine }}=3.20, C N R_{\text {gadolinium }}=2.80\right)$ than gold and gadolinium $\left(C N R_{\text {gadolinium }}=1.67, C N R_{\text {gold }}=1.37\right)$.

Conclusions: SPCCT enables multicolour quantitative imaging. As a result, it should be possible to perform imaging of multiple uptake phases of a given tissue/organ within a single scan by injecting different contrast agents sequentially.
\end{abstract}

Keywords: Gadolinium, Gold, lodine, Phantoms (imaging), Tomography (x-ray computed)

\section{Key points}

- SPCCT is capable of discriminating between two contrast agents within the same image location via K-edge imaging.

- SPCCT K- enables the discrimination between either one K-edge material and iodine such as gadolinium, or two K-edge materials, such as gold and gadolinium.

- SPCCT can qualitatively and quantitatively separate either gadolinium and iodine or gadolinium and gold

\footnotetext{
* Correspondence: salimaymeric@gmail.com; salim.si-mohamed@chu-lyon.fr ${ }^{1}$ University Claude Bernard Lyon1, CREATIS, CNRS UMR 5220, INSERM U1206, INSA-Lyon, Lyon, France

${ }^{2}$ Radiology Department, Hospices Civils de Lyon, Lyon, France

Full list of author information is available at the end of the article
}

with good accuracy (offsets between -0.68 and $\left.0.89 \mathrm{mg} / \mathrm{mL}, \mathrm{R}^{2} \geq 0.97\right)$.

\section{Background}

Since the emergence of clinical dual-energy computed tomography (DECT) and spectral photon-counting computed tomography (SPCCT), there has been an increasing interest in using these systems to discriminate between different contrast agents, based on their specific x-ray attenuation characteristics [1-9]. For example, DECT has recently been used for in vivo depiction of the small bowel wall and for the differentiation between a vascular and an enteric injury, using a combination of an intravenous iodinated contrast agent with an oral tantalum- or tungsten-based contrast agent [10] or oral bismuth [11]. However, because DECT is 
limited by its sampling capacity of only two predefined energy spectra, typically high $(140 \mathrm{kVp})$ and low $(80 \mathrm{kVp})$ $[5,12,13]$, the attenuation model used for reconstruction is restrained to the material decomposition of only two base images [5, 14]. Any other material is represented as an equivalent ratio of the two base materials. Moreover, the DECT material decomposition scheme does not contain the K-edge discontinuity, which has to be determined using data from an additional energy $[5,15,16]$.

Unlike DECT, SPCCT uses energy-resolving detectors that can simultaneously sample the energy spectrum at multiple regions. This allows a higher spectral resolution, enabling the identification of material-specific spectral characteristics, such as the K-edge signature of the contrast agent [5, 15-18]. This is particularly interesting as the K-edge energies of contrast agents that contain heavy elements such as gadolinium, ytterbium, bismuth or gold are within the clinical $x$-ray tube spectrum $[1,17,19-21]$. The material decomposition can then be extended to include the K-edge of the contrast agents as a separate basis, allowing for multicolour imaging [8, 22, 23]. However, despite its high spectral resolution, it is still unknown whether the SPCCT system can distinguish and quantify two contrast agents when they are mixed within the same volume (either one with high and the other with low K-edge energy or both with high K-edge energies).

In this study, we proposed to fill this gap in knowledge by performing multicolour imaging with SPCCT and detect as well as quantify iodine, gadolinium and gold in different mixtures, with a single scan.

\section{Methods}

\section{Phantom preparation}

A custom-made polyoxymethylene cylindrical phantom with a diameter of $13 \mathrm{~cm}$ and 12 holes with a $1.5-\mathrm{cm}$ diameter was used. The contrast agents used in this study were: gold nanoparticles (AuNP; $65 \mathrm{mg} / \mathrm{mL}$, size $18 \mathrm{~nm}$, synthesised in-house) [24]; an iodinated contrast agent (Iomeron $400 \mathrm{mg} / \mathrm{mL}$, Bracco, Milan, Italy); and a gadolinium chelate (MultiHance $0.5 \mathrm{mmoL} / \mathrm{mL}, 78.625 \mathrm{mg} / \mathrm{mL}$, Bracco, Milan, Italy). The samples were loaded into the phantom using $1.5-\mathrm{mL}$ polypropylene centrifuge tubes. Two sets of 11 tubes were prepared, each containing two contrast agents diluted in phosphate buffer saline (PBS) and mixed in varying proportions, as previously described $[8,21]$ (Fig. 1c, d). The mixed agents were either gadolinium mixed with iodine or gadolinium mixed with gold. Eleven samples each of unmixed AuNP, gadolinium or iodine were also prepared in order to examine the effects of mixing the contrast agents on the measurement of their concentrations. The proportions of each pair of contrast agents were adjusted using a proper MATLAB code that allows calculations based on data obtained from imaging unmixed contrast agents. The concentrations $(\mathrm{mg} / \mathrm{mL})$ of the mixtures depended on the corresponding attenuation at $120 \mathrm{kVp}$. The target attenuation for each solution was chosen within the clinical standard for CT angiography applications, i.e. 280 Hounsfield units (HU) approximately [25]. Hence, the concentrations of gadolinium, AuNP and iodine were in the range of 0 $7.45 \mathrm{mg} / \mathrm{mL}, 0-10.4 \mathrm{mg} / \mathrm{mL}$ and $0-8 \mathrm{mg} / \mathrm{mL}$, respectively. The contrast agent concentrations are presented in Table 1.

\section{Spectral photon-counting computed tomography}

The modified clinical base, small field of view (FOV) SPCCT prototype system (Philips Healthcare, Haifa, Israel) is equipped with a conventional $\mathrm{x}$-ray tube that can be set with a tube voltage at 80,100 or $120 \mathrm{kVp}$ and tube current values in the range of $10-100 \mathrm{~mA}$. The system is based on a semi-conductor detector technology operated in single photon-counting mode with energy discrimination $[15,26]$. The system is operated as a nine-row scanner with an effective $\mathrm{z}$-collimation of about $2.5 \mathrm{~mm}$ in the isocentre and an imaging FOV of $168 \mathrm{~mm}$ in-plane. Axial scans $>360^{\circ}$ were performed at a tube current of $100 \mathrm{~mA}$ and a tube voltage of $120 \mathrm{kVp}$ (weighted CT dose index $=0.74 \mathrm{mGy}$ ) with a scanner rotation time of $1 \mathrm{~s}$ and 2400 projections per rotation. Note that the CT dose index on SPCCT prototype is higher than what we expect on a future clinical scanner for the same $\mathrm{kVp}$ and $\mathrm{mAs}$ because the current creates very small z-collimation resulting in low dose efficiency due to the small useful part of the beam relative to the penumbra part. Each pixel in each projection reading consisted of a group of five integer numbers, representing the number of crossings (from low to high) of the analogue pulse signal of predefined energy thresholds. For low flux and negligible pile-up, these numbers corresponded to the counts in single-ended pulse-height windows that resulted from the convolution of the $\mathrm{x}$-ray spectrum and the detector response function. Due to the small FOV and the small z-collimation of the prototype, scatter effects were much smaller than for clinical CT. Thus, the detector of this prototype was not equipped with an anti-scatter grid. This system had five thresholds that could be adjusted in order to allow photon energy-based discrimination of an element. In the cases of gadolinium and gold, we set two thresholds just below and above their K-edges, at $50.2 \mathrm{keV}$ and $80.7 \mathrm{keV}$, respectively, as was detailed in the study by Roessl et al. [27]. One additional energy threshold served as a noise threshold and was set to $30 \mathrm{keV}$. Hence, the energy thresholds were set at 30,51, 64, 72 and $85 \mathrm{keV}$ for the gadolinium study, at 30,53, 78, 83 and $98 \mathrm{keV}$ for the gold study, at 30, 51, 78, 83 and $98 \mathrm{keV}$ for the gold and gadolinium mixture study, and at $30,51,64,72$ and $85 \mathrm{keV}$ for the iodine and gadolinium mixture study (Fig. 1a, b). The energy thresholds for the iodine study were set at 30, 51, 64, 72 and $85 \mathrm{keV}$, even though iodine could not be efficiently detected by the K-edge technique due to the low numbers of photons around its K-edge energy (33.2 keV) [28]. 


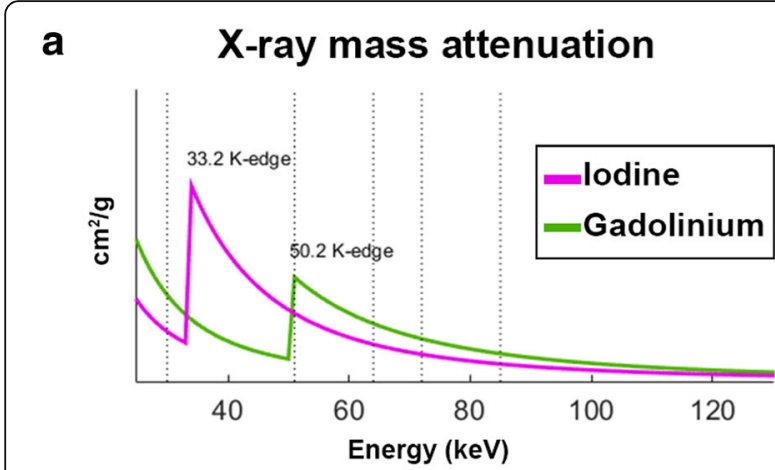

\section{c Phantom with mixtures}

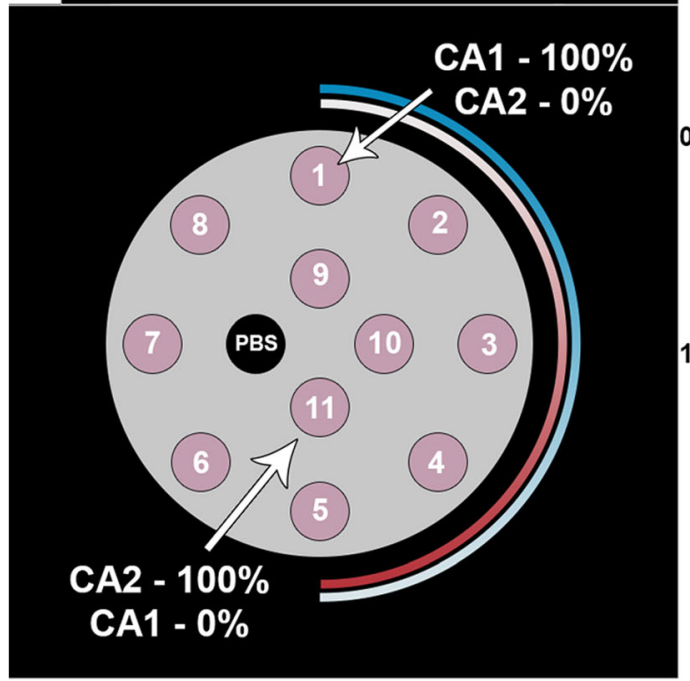

b X-ray mass attenuation

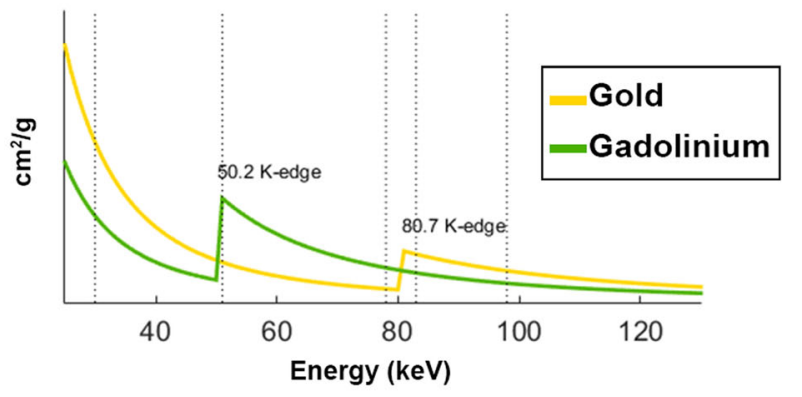

d

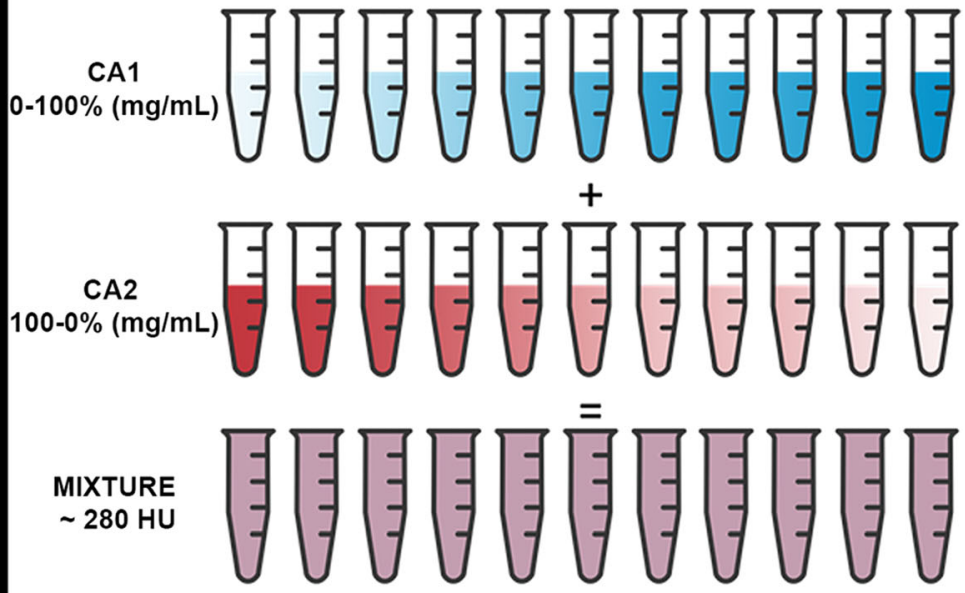

Fig. 1 Representation of the K-edge energies and thresholds (dotted lines) used for the characterisation of each element. x-ray mass attenuations of iodine (purple) and gadolinium (green) (a), as well as gold (yellow) and gadolinium (green) (b) are shown with solid lines. c, $\mathbf{d}$ Eleven tubes of mixed contrast agents were prepared. The solutions were organised in a spiral, such that contrast agent 1 (in blue) was prepared at a decreasing concentration while contrast agent 2 (in red) was prepared at an increasing concentration. The tubes in the phantom have the same colour on the schema because of their same-targeted CT attenuation values ( $280 \mathrm{HU})$

Data acquisition and spectral image reconstruction

All solutions were scanned in the same phantom using the SPCCT prototype. The same SPCCT scan protocol was used, i.e. axial scan at a tube voltage of $120 \mathrm{kVp}$, tube current of $100 \mathrm{mAs}$ and FOV of $160 \mathrm{~mm}$. For each pixel, a maximum likelihood estimator was used to derive an equivalent water-thickness per pixel from the photon counts in the five energy bins. Conventional images were then reconstructed from the water-thickness equivalent sinograms using a filtered back-projection algorithm. A material decomposition based on a forward projection model (per detector) and maximum likelihood, and literature data provided by the National Institute of Standards and Technology (Gaithersburg, MD, USA) [29], was used for the energy-dependent attenuation of the base materials. For each phantom configuration, a specific material basis consisting of at least water and iodine and-in case of their presence-gadolinium and/or gold was chosen. For example, a three-material water-iodine-gadolinium basis was used for the gadolinium-iodine study while a four-material water-iodine-gold-gadolinium basis was selected for the gadolinium-gold mixture study. The five multi-energy sinograms were then decomposed with a maximum-likelihood algorithm [16] into a set of up to four base material sinograms for the given material basis. Finally, the base material sinograms were individually reconstructed. The sinograms were individually reconstructed with a wedge reconstruction algorithm [30, 31] on a $0.4 \times 0.4 \times 0.25 \mathrm{~mm}^{3}$ voxel grid. The reconstructed volume was then averaged over all eight slices to reduce noise so that the images analysed were $2-\mathrm{mm}$ thick. Hence, for each of the contrast agent mixtures, a distinct set of contrast material images appropriate for each contrast agent combination was generated within a few minutes for each slice, i.e. water, iodine, gadolinium and gold images. The water and iodine images were 
Table 1 Concentrations of the three different contrast agents used in mixtures or individually diluted to obtain the six sets of 11

\begin{tabular}{|c|c|c|c|c|c|c|c|}
\hline \multirow{2}{*}{$\begin{array}{l}\text { Tube } \\
\text { ID }\end{array}$} & \multicolumn{2}{|c|}{ Mixed gold and gadolinium } & \multicolumn{2}{|c|}{ Mixed iodine and gadolinium } & \multirow{2}{*}{$\begin{array}{l}\text { Gold only } \\
\text { Gold } \\
(\mathrm{mg} / \mathrm{mL})\end{array}$} & \multirow{2}{*}{$\begin{array}{l}\text { Gadolinium only } \\
\text { Gadolinium } \\
(\mathrm{mg} / \mathrm{mL})\end{array}$} & \multirow{2}{*}{$\begin{array}{l}\text { lodine onl) } \\
\text { lodine } \\
(\mathrm{mg} / \mathrm{mL})\end{array}$} \\
\hline & Gold (mg/mL) & $\begin{array}{l}\text { Gadolinium } \\
(\mathrm{mg} / \mathrm{mL})\end{array}$ & $\begin{array}{l}\text { lodine } \\
\text { (mg/mL) }\end{array}$ & $\begin{array}{l}\text { Gadolinium } \\
(\mathrm{mg} / \mathrm{mL})\end{array}$ & & & \\
\hline 1 & 0 & 7.46 & 8 & 0 & 0 & 0 & 8 \\
\hline 2 & 0.98 & 6.67 & 7.2 & 0.79 & 0.98 & 0.79 & 7.2 \\
\hline 3 & 1.95 & 5.89 & 6.4 & 1.57 & 1.95 & 1.57 & 6.4 \\
\hline 4 & 3.25 & 5.1 & 5.6 & 2.36 & 3.25 & 2.36 & 5.6 \\
\hline 5 & 4.22 & 4.32 & 4.8 & 2.75 & 4.22 & 2.75 & 4.8 \\
\hline 6 & 5.2 & 3.53 & 4 & 3.53 & 5.2 & 3.53 & 4 \\
\hline 7 & 6.17 & 2.75 & 3.2 & 4.32 & 6.17 & 4.32 & 3.2 \\
\hline 8 & 7.15 & 2.36 & 2.4 & 5.1 & 7.15 & 5.1 & 2.4 \\
\hline 9 & 8.13 & 1.57 & 1.6 & 5.89 & 8.13 & 5.89 & 1.6 \\
\hline 10 & 9.43 & 0.79 & 0.8 & 6.67 & 9.43 & 6.67 & 0.8 \\
\hline 11 & 10.4 & 0 & 0 & 7.07 & 10.4 & 7.07 & 0 \\
\hline
\end{tabular}

reconstructed for each solution no matter the content; the gadolinium and gold images were reconstructed only in the presence of the material. The images were then analysed with further post-processing using deringing and smoothing of the contrast material images with a Gaussian kernel with a 2-pixel radius and sigma.

\section{Image analysis}

Image analysis was performed using MATLAB (R2015a, MathWorks, Inc.). The samples were automatically detected on conventional images and circular regions of interest (ROIs) were automatically drawn in the middle of each tube. The same ROIs were used on all images generated on a given dataset and mean and standard deviation were computed for each ROI. Linear regression was performed between the prepared and measured concentrations for all solutions. The cross-contamination of the material decomposition, i.e. the amount of a measured material in other material image (e.g. amount of gadolinium and gold measured in water and iodine images), was measured for each material as the root mean square error (RMSE) of its concentration in the other basis materials' ROIs, where no signal would be expected in the iodine image and where a mean value of $1000 \mathrm{mg} / \mathrm{mL}$ would be expected in the water image.

For each contrast agent in both unmixed and mixed solutions, the CNR relative to a homogeneous PBS tube was calculated according to the following equation:

\section{Statistical analysis}

In order to assess the impact of mixing the contrast agents, a reliability analysis between the non-mixed and the mixed measured concentrations was performed using intraclass correlation coefficients (ICC) and their 95\% confident intervals (CI). The statistical analyses were performed using SPSS statistical package version 24 (SPSS Inc., Chicago, IL, USA) based on a mean rating for each contrast agent ( $k=3$ e.g. Gd, Gd + AuNP, Gd + I), two-way mixed-effects model.

\section{Results}

As expected from the experimental design, different solutions of mixed contrast agents could not be differentiated on conventional images as all tubes had similar attenuations $(279 \pm 10 \mathrm{HU})$. However, we found that the contrast agents were accurately identified in the material images and that the water images showed only the material-based water map in the solutions and in the phantom (Fig. 2).

The correlations between the measured and prepared concentrations were strongly linear for all dilutions (all $R^{2} \geq 0.97$ ). The slopes were close to 1 for the unmixed contrast agents solutions $(0.98 \leq$ slope $\leq$ 1.04), but were underestimated for the mixed contrast agents solutions $(0.81 \leq$ slope $\leq 0.95)$ (Figs. 3 and 4$)$. The underestimations were slightly more marked for

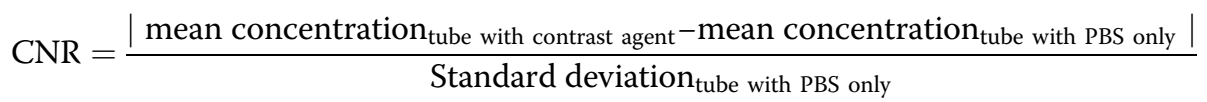




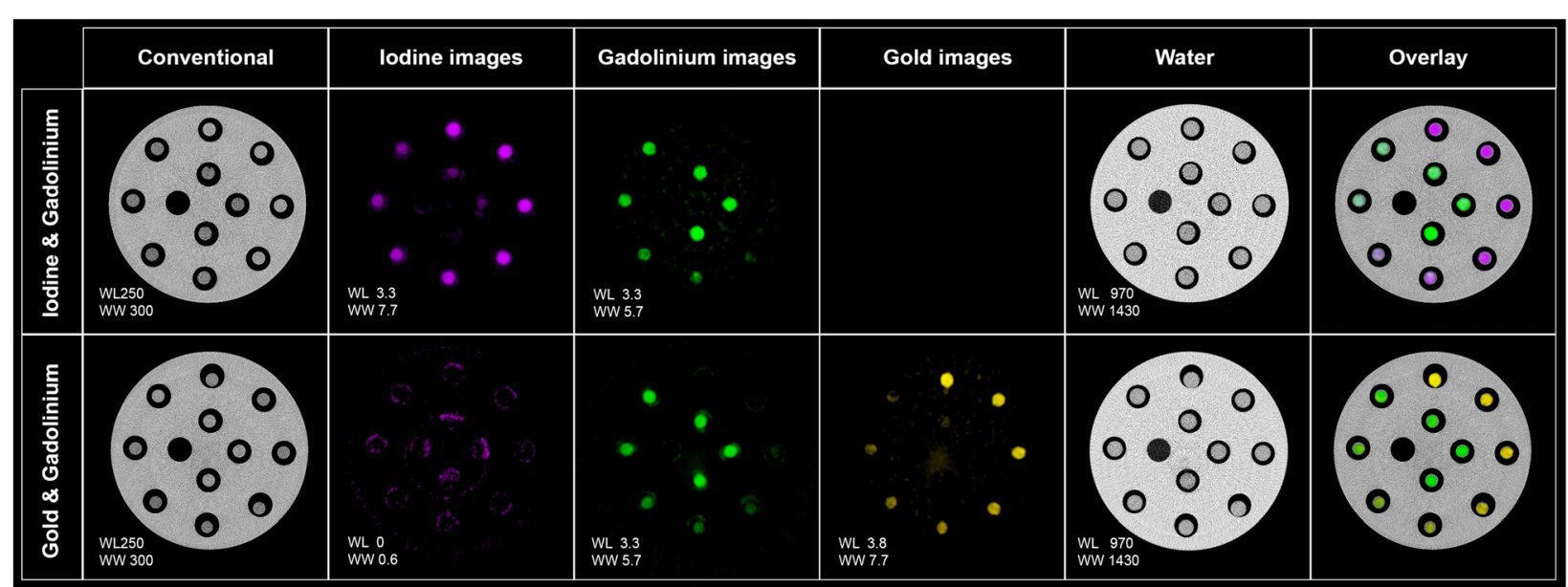

Fig. 2 Spectral photon-counting images of conventional, iodine (purple), gadolinium (green), gold (yellow) and water for both sets of mixed contrast agents. Top: iodine-gadolinium mixture; bottom: gold-gadolinium mixture; conventional image units are HU and material images (water, iodine, gold, gadolinium) units are $\mathrm{mg} / \mathrm{mL}$. Note that no gold image was generated from the material decomposition process for the iodine-gadolinium mixture, whereas an iodine image was generated from the material decomposition process for the gadolinium-gold mixture

the gadolinium-gold mixture (gadolinium slope 0.81; gold slope 0.90), than for the iodine-gadolinium mixture (gadolinium slope 0.85; iodine slope 0.95) (Fig. 6). Additionally, the offset values were very low for single contrast agent solutions $(-0.13 \leq$ offset $\leq 0.22 \mathrm{mg} / \mathrm{mL})$, but most of the offset values were significantly different from zero when the contrast agents were mixed $(-0.68 \leq$ offset $\leq 0.89 \mathrm{mg} / \mathrm{mL}$ ) (Table 2 and Fig. 4).
The ICC analysis showed a significant linear relation between prepared and measured concentrations indicating an excellent degree of reproducibility between each of the contrast agent measurements (Table 2). Importantly, the linear relation indicates that offsets observed when contrast agents were mixed are not concentration-dependent. These quantitative results are summarised in Tables 2 and 3.

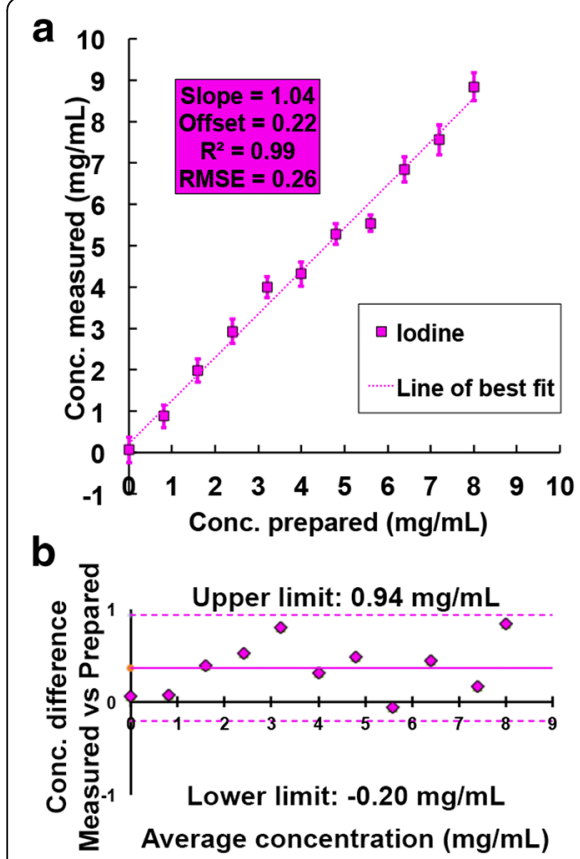

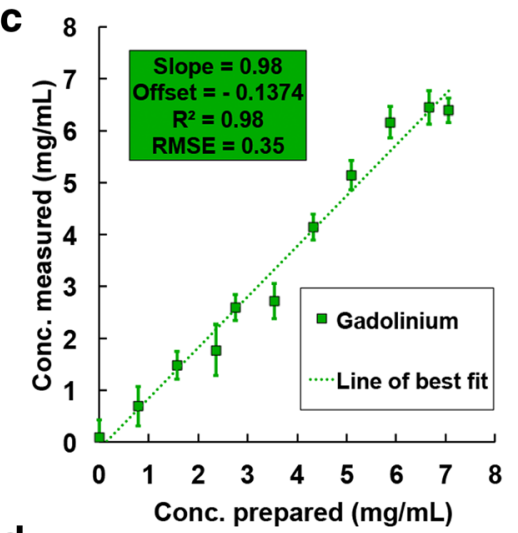

d

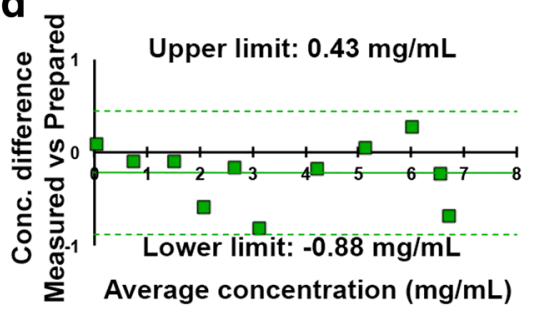

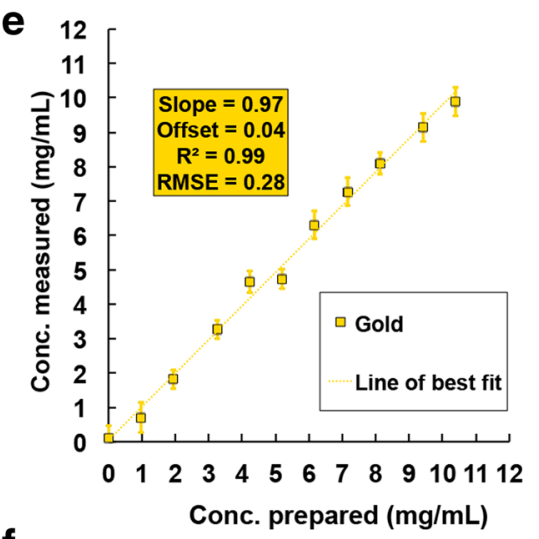

f

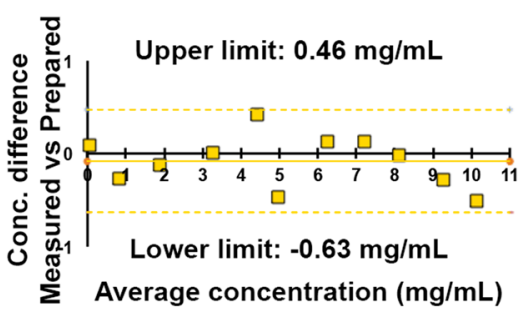

Fig. 3 Measurements of the concentrations of contrast agents in the unmixed solutions. The concentrations of iodine (purple) (a, b), gadolinium (green) (c, d) and gold (yellow) (e, f) were measured in their respective unmixed solutions. a, c, e Linear regression; b, $\mathbf{d}$, $\mathbf{f}$ Bland-Altman analysis. Note the linear correlations and the slope values approaching 1 for all contrast agents 
a
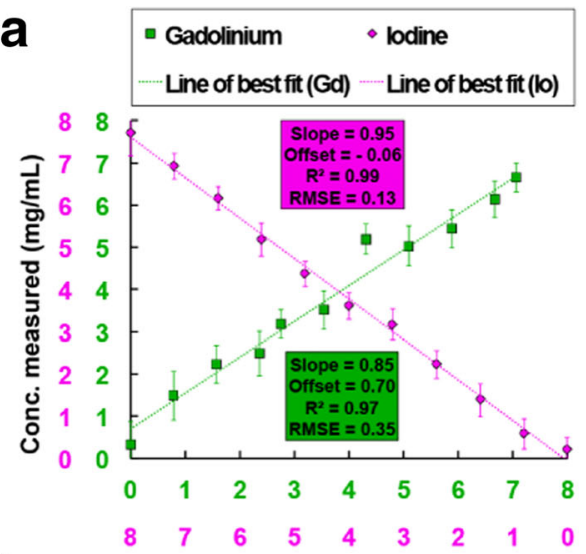

b Conc. prepared $(\mathrm{mg} / \mathrm{mL})$

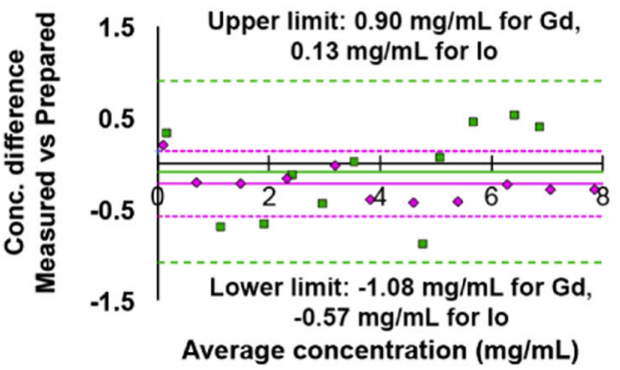

C

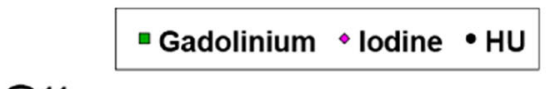

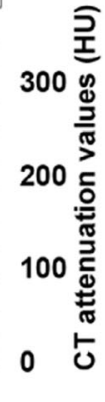
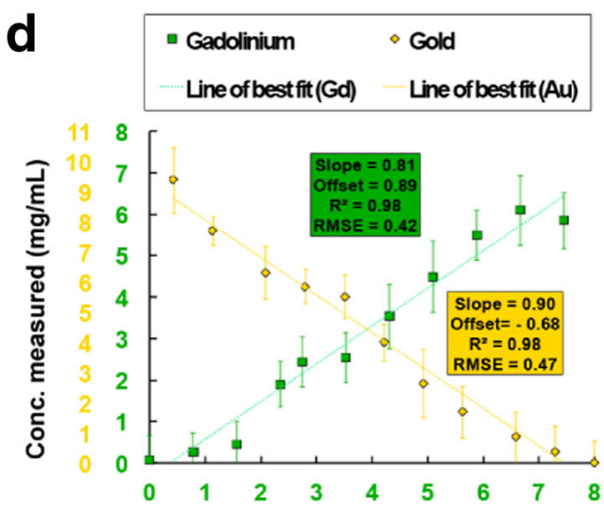

$\mathbf{e}$

Conc. prepared $(\mathrm{mg} / \mathrm{mL})$

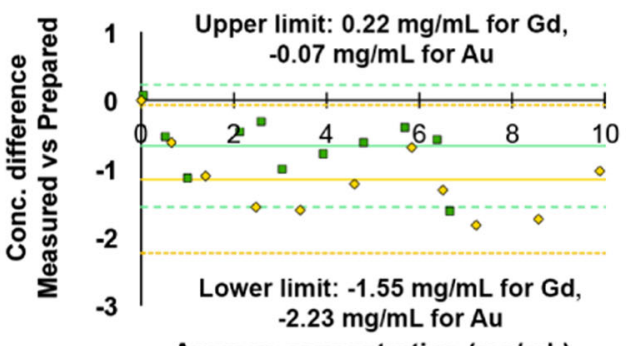

Average concentration $(\mathrm{mg} / \mathrm{mL})$

f
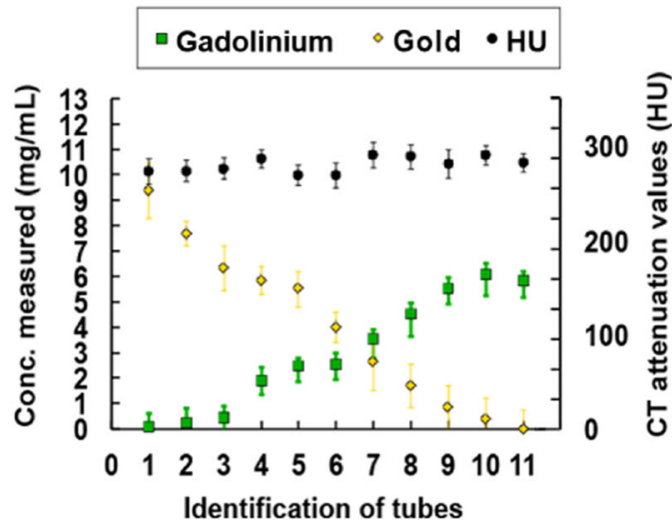

Fig. 4 Measurements of the concentrations of contrast agents and attenuation values in the mixed solutions. The concentrations of each contrast agent were measured within the gadolinium-iodine $(\mathbf{a}, \mathbf{b}, \mathbf{c})$ and gadolinium-gold $(\mathbf{d}, \mathbf{e}, \mathbf{f})$ mixtures. $\mathbf{a}$, $\mathbf{d}$ Linear regression; $\mathbf{b}$, e: Bland-Altman analysis; $\mathbf{c}, \mathbf{f}$ : graphs of the mean \pm standard deviation of attenuation values and concentrations. Note that, as expected, the measured concentrations in the tubes varied inversely between the two mixed contrast agents

Table 2 Linear regression coefficients of each reconstructed image from the different contrast agent mixtures and of the only individual contrast agent solutions

\begin{tabular}{|c|c|c|c|c|c|c|c|}
\hline \multirow[t]{2}{*}{ Image } & \multicolumn{2}{|c|}{ Mixed gold and gadolinium } & \multicolumn{2}{|c|}{ Mixed iodine and gadolinium } & \multirow{2}{*}{$\begin{array}{l}\text { Gold only } \\
\text { Gold }\end{array}$} & \multirow{2}{*}{$\begin{array}{l}\text { Gadolinium only } \\
\text { Gadolinium }\end{array}$} & \multirow{2}{*}{$\begin{array}{l}\text { lodine only } \\
\text { lodine }\end{array}$} \\
\hline & Gold & Gadolinium & lodine & Gadolinium & & & \\
\hline Slope & 0.90 & 0.81 & 0.95 & 0.81 & 0.97 & 0.98 & 1.04 \\
\hline Offset & -0.68 & 0.89 & -0.06 & 0.88 & 0.04 & -0.13 & 0.22 \\
\hline$R^{2}$ & 0.98 & 0.98 & 0.99 & 0.98 & 0.99 & 0.98 & 0.99 \\
\hline RMSE & 0.47 & 0.42 & 0.13 & 0.35 & 0.28 & 0.35 & 0.26 \\
\hline $95 \% \mathrm{Cl}$ & $0.83-1$ & $0.86-1.1$ & $0.96-1$ & $0.77-1$ & $0.9-0.97$ & $0.82-1$ & $0.99-1$ \\
\hline
\end{tabular}

RMSE root mean square error, $\mathrm{Cl}$ confidence interval 
The water images did not show any cross-contamination for either the unmixed or the mixed solutions, as confirmed by the very low RMSE values (RMSE $\leq 0.08 \mathrm{mg} / \mathrm{mL}$ ). Similarly, the iodine images did not show any cross-contamination for the unmixed solutions, despite a RMSE at $0.33 \mathrm{mg} / \mathrm{mL}$ for the gadolinium dilutions due to negative iodine measured concentrations. Conversely, the iodine images showed some cross-contamination for the gold-gadolinium mixture, as confirmed by a RMSE at $0.34 \mathrm{mg} / \mathrm{mL}$ and by the measured concentrations of iodine. The cross-contamination in iodine images increased with increasing concentrations of gadolinium but not gold (Table 3 and Fig. 5).

The CNR values observed for each contrast agent were higher for the unmixed solutions, in particular for the gold-gadolinium mixture (unmixed gold CNR slope 2.80; unmixed gadolinium CNR slope 3.11; gold-gadolinium mixture: gold CNR slope 1.37, gadolinium CNR slope 1.67) (Fig. 6). Furthermore, the CNR slope for unmixed iodine was better than for the gadolinium-iodine mixture (unmixed iodine CNR slope 3.45; gadoliniumiodine mixture: iodine CNR slope 3.20).

\section{Discussion}

CT is an imaging modality with an excellent spatial resolution and fast acquisition times that has laid the foundation for SPCCT. In the present study, we demonstrate that SPCCT has the ability to separate two mixed contrast agents qualitatively and quantitatively. Indeed, the SPCCT energy-resolving detectors enable such spectral resolution that it is possible to distinguish K-edge signatures of different contrast agents, within the same voxel and at the same time. Consequently, separate quantity maps for each individual contrast agent can be generated. In addition, while it has been previously shown that SPCCT can be used for absolute quantification of unmixed multiple contrast agents in vivo [7, 8, 21-23, 32,33 ], few studies have investigated the accuracy of the quantification [21, 33].

Recent in vitro studies have reported the quantification of iodine and gadolinium contrast agents using DECT $[34,35]$. However, this technology can decompose the attenuations into at most two components because it can perform only two measurements per pixel. SPCCT, on the other hand, provides five photon-count measurements (five bins) per pixel, which theoretically allows the concomitant decomposition of the attenuations on a two- to four-material basis; two non-K-edge materials (e.g. water and iodine), and one or two K-edge materials (e.g. gadolinium and gold), given that their K-edge energies are in two different energy bins. Our results confirm this theory. In fact, we were able to show an accurate affine response of the material decomposition process both for unmixed (iodine, gadolinium and gold) and mixed (iodine-gadolinium and gadolinium-gold mixtures) solutions, despite slightly shifted offsets. Nevertheless, when two contrast agents were mixed, we observed an underestimation of the quantification of the contrast agents that was more marked for K-edge materials (e.g. gold and gadolinium). Similarly, mixing the two K-edge materials led to some cross-contamination in the iodine image. Another consequence of mixing the materials was the increase of noise and the decrease of the CNR of the materials, much more adversely affected for the K-edge materials, i.e. gadolinium and gold. Indeed, as a result we observed a twofold decrease of the CNR for the mixture of gadolinium and gold demonstrating that the four-material basis approach comes with a lower sensitivity performance limiting drastically the trade-off between contrast and noise. These outcomes can be explained by the fact that including more components in the material decomposition process means higher degrees of freedom for the maximum likelihood algorithm, leading to smaller signal per component and therefore more noise and more noise-induced bias. In addition, we observed that among the K-edge materials, the gadolinium image was more contrasted than the gold one. This can be explained by the fact that gadolinium beneficiates from a better balance of photons below and above its K-edge energy than gold in a $120-\mathrm{kVp}$ beam, leading to a more accurate material decomposition.

The origins of all these imperfections can be explained by the noise on the photon counts, which propagates to noise in the decomposed sinograms and ultimately to the material maps. In addition to the inherent quantum noise, physical effects such as pulse pile-up and charge sharing as well as inaccuracies in the spectral model of the system (e.g. x-ray tube spectrum, detector response function) will further degrade the quantification results if not properly addressed in the forward model which is solved by the maximum likelihood decomposition [1, 16]. The decomposition and reconstruction algorithms are still

Table 3 Root mean square error (RMSE) coefficients of water and iodine reconstructed images from the different unmixed and mixed solutions

\begin{tabular}{|c|c|c|c|c|c|c|c|c|}
\hline \multirow[t]{2}{*}{ Image } & \multicolumn{2}{|c|}{ Mixed gold and gadolinium } & \multirow{2}{*}{$\begin{array}{l}\text { Mixed iodine and gadolinium } \\
\text { Water }\end{array}$} & \multicolumn{2}{|c|}{ Gadolinium } & \multicolumn{2}{|l|}{ Gold } & \multirow{2}{*}{$\begin{array}{l}\text { lodine } \\
\text { Water }\end{array}$} \\
\hline & Water & lodine & & Water & lodine & Water & lodine & \\
\hline RMSE & 0.08 & 0.34 & 0.01 & 0.01 & 0.33 & 0.01 & 0.18 & 0.01 \\
\hline
\end{tabular}



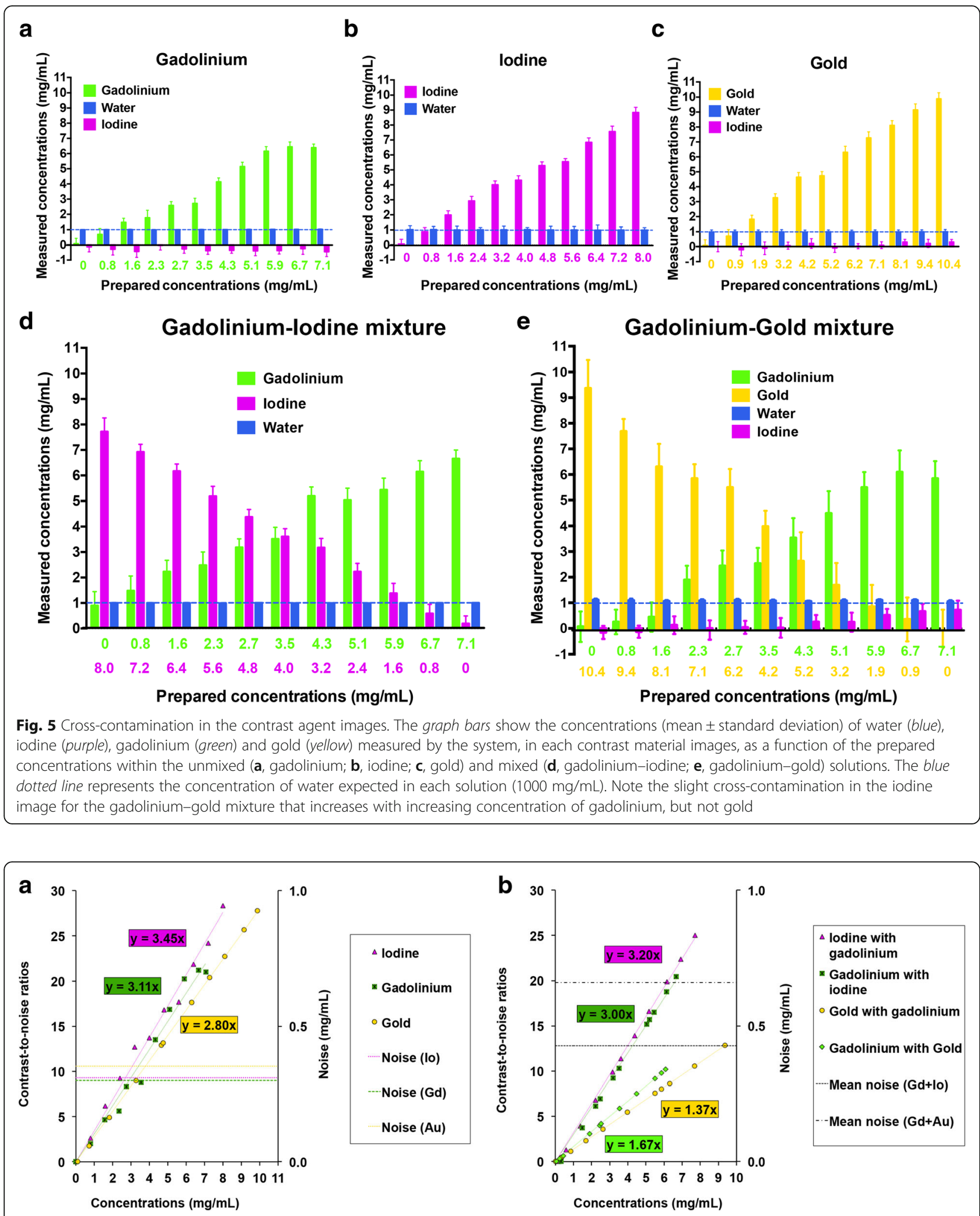

Fig. 6 CNRs of the contrast agents. The graphs represent the CNR values depending on the concentration of the contrast agent and the noise values of the contrast agents measured in their respective contrast material maps within the unmixed (a) and mixed (b) solutions. The dotted lines represent the noise measured in a tube filled with PBS only within the contrast material images. Mean noise is the mean of the noise measured within the two contrast material images of each mixture 
under development. Quantification improvements are expected as more accurate models and further noise handling algorithms will be applied. For example, researchers are actively looking into including some of the still un-modelled physical effects and enhancing the accuracy of the incident spectrum and detector response function [36, 37]. Additionally, mitigating the noise would probably require using regularised iterative methods, either during the material decomposition process [38], during the reconstruction process [39] or in a one-step algorithm that would embed both processes [40].

Based on our results, and the fact that all SPCCT reconstructed images have a 100\% spatial registration [13], using multiple contrast agents with different pharmacokinetics in the same biological system simultaneously can reasonably be considered. Several applications of such methods in the vascular system can be considered. For example, different contrast agents injected sequentially within a single scan could allow the imaging of multiple uptake phases of a given tissue/organ [7, 32]. It could also be possible to simultaneously visualise the vascular lumen and wall in pathologies such as atherosclerosis by using a combination of specific and non-specific contrast agents [22]. These applications would considerably enhance the diagnostic capabilities and decrease the patients' exposure to radiation. Finally, for the past 20 years, increasing efforts have been directed toward in vivo imaging of gene expression and enzyme activity, particularly in oncology research. It would be interesting to develop new multi-atom contrast agents that could be modified or cleaved in targeted tissues to allow cellular activity imaging [41-43].

Today, however, the simultaneous use of multiple intravascular heavy metal-based contrast agents is still not clinically approved. Moreover, none of the K-edge candidates are approved for human use, i.e. the heavy atoms, even in the case of contrast agent-based nanoparticles. As a consequence, further pharmacological evaluations of currently available agents (e.g. concomitant use of iodine and gadolinium) or the development of new contrast media are needed.

As a study limitation, we should note that the work we report here has been done using relatively simple phantoms and a preclinical SPCCT scanner. In order to extend our study to clinical applications, we are now using a machine scaled for clinical use and assessing its spectral capabilities under varying conditions (tube currents and voltage) with phantom conditions closer to human characteristics (size, attenuation, FOV).

In conclusion, the SPCCT prototype used in this study can qualitatively and quantitatively differentiate multiple contrast agents within the same solution, opening perspectives in clinical applications. However, to fully take advantage of the SPCCT multicolour imaging performance, new agents containing K-edge materials must be developed.

\section{Abbreviations \\ AuNP: Gold nanoparticles; Cl: Confidence interval; CNR: Contrast-to-noise ratio; DECT: Dual-energy computed tomography; FOV: Field of view; HU: Hounsfield units; ICC: Intraclass correlation coefficient; PBS: Phosphate buffered saline; RMSE: Root mean square error; ROI: Region of interest; SPCCT: Spectral photon-counting computed tomography}

\section{Availability of data and materials}

The authors declare that data will not be shared publicly.

\section{Funding}

This work was supported by European Union Horizon 2020 grant No 643694, France Life Imaging (FLI) and NIH (R01 HL131557).

\section{Acknowledgements}

We thank employees from the CERMEP-Imagerie du Vivant for helping with the experiment. We also thank Dr. Ira Blevis, Dr. Simon Rit, Dr. Cyril Mory, Dr. Yaron Younes, Dr. Luisa Poggi and Dr. Joelle Belgamire for their help in designing and writing the manuscript.

\section{Authors' contributions \\ SSM, DB, MS and PD conceived and designed the experiments. SSM and DB performed the experiments. SSM and DB analysed the data. SSM, DB, MS, LB, $P D, P C, Y Y, M R, Y Y, V T L, D P C, P N, E R$ and $A A$ contributed reagents/materials/ analysis tools. All authors contributed to the writing of the manuscript. All authors read and approved the final manuscript.}

Ethics approval and consent to participate

Not applicable.

\section{Consent for publication}

Not applicable

\section{Competing interests}

Philippe Coulon, Michal Rokni, Ewald Roessl, Yoad Yagil and Ami Altman are employees of Philips Healthcare, the manufacturer of the scanner.

\section{Publisher's Note}

Springer Nature remains neutral with regard to jurisdictional claims in published maps and institutional affiliations.

\section{Author details}

${ }^{1}$ University Claude Bernard Lyon1, CREATIS, CNRS UMR 5220, INSERM U1206, INSA-Lyon, Lyon, France. ${ }^{2}$ Radiology Department, Hospices Civils de Lyon, Lyon, France. ${ }^{3}$ Global Advanced Technologies, CT, Philips, Haifa, Israel. ${ }^{4}$ Department of Radiology, University of Pennsylvania, Philadelphia, PA, USA. ${ }^{5} \mathrm{CT}$ Clinical Science, Philips, Suresnes, France. ${ }^{6}$ Philips GmbH Innovative Technologies, Research Laboratories, Hamburg, Germany.

Received: 30 May 2018 Accepted: 3 August 2018

Published online: 17 October 2018

\section{References}

1. Schlomka JP, Roessl E, Dorscheid R et al (2008) Experimental feasibility of multi-energy photon-counting K-edge imaging in pre-clinical computed tomography. Phys Med Biol 53:4031-4047

2. Cole LE, Ross RD, Tilley JM, Vargo-Gogola T, Roeder RK (2015) Gold nanoparticles as contrast agents in $\mathrm{x}$-ray imaging and computed tomography. Nanomedicine (Lond) 10:321-341

3. Hong SR, Chang S, Im DJ et al (2016) Feasibility of single scan for simultaneous evaluation of regional krypton and iodine concentrations with dual-energy CT: an experimental study. Radiology 281:597-605

4. Altman A, Carmi R (2009) TU-E-210A-03: a double-layer detector, dualenergy CT-principles, advantages and applications. Med Phys 36:27502750 
5. McCollough CH, Leng S, Yu L, Fletcher JG (2015) Dual- and multi-energy CT: principles, technical approaches, and clinical applications. Radiology 276: 637-653

6. Mongan J, Rathnayake S, Fu Y et al (2012) In vivo differentiation of complementary contrast media at dual-energy CT. Radiology 265:267-272

7. Symons R, Krauss B, Sahbaee P et al (2017) Photon-counting CT for simultaneous imaging of multiple contrast agents in the abdomen: an in vivo study. Med Phys 44:5120-5127

8. Cormode DP, Si-Mohamed S, Bar-Ness D et al (2017) Multicolor spectral photon-counting computed tomography: in vivo dual contrast imaging with a high count rate scanner. Sci Rep 7:4784

9. Si-Mohamed S, Bar-Ness D, Sigovan M et al (2017) Review of an initial experience with an experimental spectral photon-counting computed tomography system. Nucl Instrum Methods Phys Res A 873:27-35 https://www.sciencedirect.com/science/article/pii/S016890021730462X

10. Rathnayake S, Mongan J, Torres AS et al (2016) In vivo comparison of tantalum, tungsten, and bismuth enteric contrast agents to complement intravenous iodine for double-contrast dual-energy CT of the bowel. Contrast Media Mol Imaging 11:254-261

11. Mongan J, Rathnayake S, Fu Y, Gao DW, Yeh BM (2013) Extravasated contrast material in penetrating abdominopelvic trauma: dual-contrast dual-energy $C T$ for improved diagnosis--preliminary results in an animal model. Radiology 268 738-742

12. Goodsitt MM, Christodoulou EG, Larson SC (2011) Accuracies of the synthesized monochromatic $C T$ numbers and effective atomic numbers obtained with a rapid kVp switching dual energy CT scanner. Med Phys 38: 2222-2232

13. Grosjean R, Sauer B, Guerra RM et al (2008) Characterization of human renal stones with mdct: advantage of dual energy and limitations due to respiratory motion. AJR Am J Roentgenol 190:720-728

14. Taguchi K, Iwanczyk JS (2013) Vision 20/20: single photon counting x-ray detectors in medical imaging. Med Phys 40:100901

15. Roessl E (2015) Imaging performance of a photon-counting computed tomography prototype. CERN, Geneva indico.cern.ch

16. Roessl E, Proksa R (2007) K-edge imaging in x-ray computed tomography using multi-bin photon counting detectors. Phys Med Biol 52:4679-4696

17. Schirra CO, Brendel B, Anastasio MA, Roessl E (2014) Spectral CT: a technology primer for contrast agent development. Contrast Media Mol Imaging 9:62-70

18. Clark DP, Badea CT (2017) Hybrid spectral CT reconstruction. PLoS One 12 e0180324

19. Pan D, Roessl E, Schlomka JP et al (2010) Computed tomography in color: nanoK-enhanced spectral CT molecular imaging. Angew Chem Int Ed Engl 49:9635-9639

20. Pan D, Schirra CO, Senpan A et al (2012) An early investigation of ytterbium nanocolloids for selective and quantitative "multicolor" spectral CT imaging. ACS Nano 6:3364-3370

21. Si-Mohamed S, Cormode DP, Bar-Ness D et al (2017) Evaluation of spectral photon counting computed tomography K-edge imaging for determination of gold nanoparticle biodistribution in vivo. Nanoscale 9:18246-18257

22. Cormode DP, Roessl E, Thran A et al (2010) Atherosclerotic plaque composition: analysis with multicolor $C T$ and targeted gold nanoparticles. Radiology 256:774-782

23. Si-Mohamed S, Thivolet A, Bonnot PE et al (2018) Improved peritoneal cavity and abdominal organ imaging using a biphasic contrast agent protocol and spectral photon counting computed tomography K-edge imaging. Investig Radiol. https://doi.org/10.1097/RLI.0000000000000483

24. Naha PC, Chhour P, Cormode DP (2015) Systematic in vitro toxicological screening of gold nanoparticles designed for nanomedicine applications. Toxicol In Vitro 29:1445-1453

25. Loewe C, Becker CR, Berletti R et al (2010) 64-slice CT angiography of the abdominal aorta and abdominal arteries: comparison of the diagnostic efficacy of iobitridol $350 \mathrm{mgl} / \mathrm{ml}$ versus iomeprol $400 \mathrm{mgl} / \mathrm{ml}$ in a prospective, randomised, double-blind multi-Centre trial. Eur Radiol 20:572-583

26. Herrmann C, Steadman R, Mülhens O (2010) ChromAIX: fast energy resolved photon-counting readout electronics for future human computed tomography. In: IEEE Nuclear Science Symposuim Medical Imaging Conference, pp 1996-1999

27. Roessl E, Herrmann C (2009) Cramér-Rao lower bound of basis image noise in multiple-energy x-ray imaging. Phys Med Biol:1307-1318
28. Roessl E, Brendel B, Engel KJ, Schlomka JP, Thran A, Proksa R (2011) Sensitivity of photon-counting based K-edge imaging in $\mathrm{x}$-ray computed tomography. IEEE Trans Med Imaging 30:1678-1690

29. Hubbell JH, Seltzer SM (2004) Tables of $x$-ray mass attenuation coefficients and mass energy-absorption coefficients. In: National Institute of Standards and Technology, Gaithersburg http://physics.nist.gov/xaamdi

30. Heuscher D, Brown K, Noo F (2004) Redundant data and exact helical conebeam reconstruction. Phys Med Biol 49:2219-2238

31. Grass M, Köhler T, Proksa R (2000) 3D cone-beam CT reconstruction for circular trajectories. Phys Med Biol 45:329-347

32. Symons R, Cork TE, Lakshmanan MN et al (2017) Dual-contrast agent photon-counting computed tomography of the heart: initial experience. Int J Cardiovasc Imaging 33:1253-1261

33. de Vries A, Roessl E, Kneepkens E et al (2015) Quantitative spectral K-edge imaging in preclinical photon-counting $x$-ray computed tomography. Invest Radiol 50:297-304

34. van Hamersvelt RW, Willemink MJ, de Jong PA et al (2017) Feasibility and accuracy of dual-layer spectral detector computed tomography for quantification of gadolinium: a phantom study. Eur Radiol 27:3677-3686

35. Pelgrim GJ, van Hamersvelt RW, Willemink MJ et al (2017) Accuracy of iodine quantification using dual energy $C T$ in latest generation dual source and dual layer CT. Eur Radiol 27:3904-3912

36. Long Y, Fessler JA (2014) Multi-material decomposition using statistical image reconstruction for spectral CT. IEEE Trans Med Imaging 33:1614-1626

37. Li Z, Leng S, Yu L, Yu Z, McCollough CH (2015) Image-based material decomposition with a general volume constraint for photon-counting CT. Proc SPIE Int Soc Opt Eng 9412. https://doi.org/10.1117/12.2082069

38. Ducros N, Abasca J, Sixou B, Rit S, Peyrin F (2017) Regularization of nonlinear decomposition of spectral x-ray projection images. Med Phys 44: e174-e187

39. Sawatzky A, Xu Q, Schirra CO, Anastasio MA (2017) Proximal ADMM for multi-channel image reconstruction in spectral x-ray CT. IEEE Trans Med Imaging 33:1657-1668

40. Schmidt T, Barber R, Sidky E (2017) A spectral CT method to directly estimate basis material maps from experimental photon-counting data. IEEE Trans Med Imaging 36:1808-1819

41. Ren G, Blum G, Verdoes M et al (2011) Non-invasive imaging of cysteine cathepsin activity in solid tumors using a 64Cu-labeled activity-based probe. PLoS One 6:e28029

42. Louie AY, Hüber MM, Ahrens ET et al (2000) In vivo visualization of gene expression using magnetic resonance imaging. Nat Biotechnol 18:321-325

43. Gaikwad HK, Tsvirkun D, Ben-Nun Y, Merquiol E, Popovtzer R, Blum G (2018) Molecular imaging of cancer using $\mathrm{X}$-ray computed tomography with protease targeted iodinated activity-based probes. Nano Lett 18:1582-1591

\section{Submit your manuscript to a SpringerOpen ${ }^{\odot}$ journal and benefit from:}

- Convenient online submission

- Rigorous peer review

- Open access: articles freely available online

- High visibility within the field

- Retaining the copyright to your article

Submit your next manuscript at $>$ springeropen.com 